\title{
Mental health resilience in offspring of depressed parents: a systematic literature review protocol
}

Eglè Padaigaitè ( $\square$ padaigaitee@cardiff.ac.uk )

Cardiff University https://orcid.org/0000-0002-5783-727X

Jessica Mayumi Maruyama

University of Sao Paulo: Universidade de Sao Paulo

Gemma Hammerton

University of Bristol

Frances Rice

Cardiff University

Stephan Collishaw

Cardiff University

\section{Research Article}

Keywords: Mental Health, Resilience, Offspring, Parents, Depression, Systematic Review

Posted Date: November 1st, 2021

DOI: https://doi.org/10.21203/rs.3.rs-502349/v1

License: (c) (i) This work is licensed under a Creative Commons Attribution 4.0 International License.

Read Full License 


\section{Abstract \\ Background}

Parental depression is associated with a range of mental health conditions and other difficulties in the offspring. Nevertheless, about one in five offspring exposed to parental depression do not develop mental health problems, indicating the presence of protective factors that may buffer parental depression-related risk effects. However, evidence of protective factors that might explain good sustained mental health in offspring of depressed parents is limited and systematic synthesis of these factors is still needed. Therefore, as far as we are aware, this will be the first systematic review that will define mental health resilience in the parental depression context and identify parental/ caregivers', child, family, and social factors associated with mental health resilience in offspring exposed to parental depression. As a secondary aim, evidence for sex-, developmental stage-, and outcome domain-specific factors associated with mental health resilience will also be examined.

\section{Methods}

A two-step search strategy will be performed. Electronic searches will be performed for articles published up to March 2021 in PsycINFO, Embase, MEDLINE, Web of Science Core Collection, and Cochrane Library. Additional articles will be identified by manually screening the references and citations of included studies. Two reviewers will independently screen titles, abstracts and full texts of articles against predetermined eligibility criteria, extract data and perform risk of bias assessments. Reviewers will be blinded to the other's decisions, and discrepancies between reviewers will be resolved during consensus meetings with a senior researcher. Results will be narratively synthesised to address primary and secondary aims.

\section{Discussion}

This systematic review will provide a comprehensive overview of protective factors associated with mental health resilience in offspring exposed to parental depression. Results will help better understand mental health resilience and factors associated with it and identify future research directions. The findings are also expected to identify targets for evidence-based prevention and intervention strategies for those in need.

\section{Systematic review registration:}

This systematic review has been registered in the International Prospective Register of Systematic Reviews (PROSPERO) database (www.crd.york.ac.uk/PROSPERO, CRD42021229955). 


\section{Background}

Adult depressive disorders and symptoms (depression) are common mental health conditions characterised by mood, somatic and cognitive symptoms that significantly affect an individual's daily functioning, including core features of anhedonia, loss of interest/ pleasure, and decreased energy [1]. Depression affects more than 264 million people worldwide and has been one of the leading causes of disability adjusted life years [2]. Depression has not only been associated with higher risk of dying by suicide [3], substance abuse [4], development of medical conditions [5, 6], and impaired social functioning [7] in individuals suffering from this condition, but also with difficulties in social relationships, education and mental health problems in their offspring $[8,9]$. Considering that up to one in five children aged 0-16 years old in the UK are exposed to parental depression and these numbers continue to rise [10], identification of factors that could improve the lives of depressed parents and their offspring remains an issue of a high public health importance [11].

Offspring of depressed parents are almost four times more likely to meet diagnostic criteria for depression compared to those of parents without a history of mental health conditions [12]. Interestingly, elevated risk of psychiatric disorders in this population is not limited to depression, and offspring of depressed parents are also more likely to suffer from a range of other mental health conditions, such as anxiety, substance use, conduct disorders, and attention deficit hyperactivity disorder (ADHD) [13, 14]. As demonstrated by Tully et al. [15], both inherited and environmental processes may be involved in the intergenerational transmission of psychopathology.

A growing body of evidence indicates that risk of psychopathology in a child may vary according to the severity and nature of parental depression. Parental depression characteristics such as severity, recurrence, and chronicity [16] and comorbid parental mental health conditions [17] have been shown to be associated with offspring psychopathology. Furthermore, depression is commonly accompanied by exposure to a range of negative life events and an increased risk of interpersonal difficulties such as marital discord [18] or parenting difficulties [19]. It has been suggested that exposure to social adversities associated with parental mental health problems may be even more influential than the parental depression itself [20].

The effect of parental depression on child's psychopathology may be also influenced by the sex of the offspring and the parent [21]. Although the majority of previously conducted studies included depressed mothers only, some studies also examined the role of fathers in shaping offspring mental health outcomes. Previous studies reported both presence [22] and absence [15] of association, or even positive father-child interaction ability to buffer the effects of maternal depression on offspring's psychopathology $[23,24]$. Therefore, it is still unclear whether parental depression effects are stronger for same-sex parent-child or mixed-sex parent-child dyads [25]. Inconsistent results could be potentially explained by 'assortative mating' [26] - a tendency for people to choose romantic partners who show similarities in personality or life experiences - that further complicates complex processes behind the intergenerational transmission of psychopathology. 
Although offspring of depressed parents are at increased risk for psychopathology, some do not develop mental health difficulties. A longitudinal study by Collishaw et al. [27] revealed that one in five adolescent offspring exposed to parental depression demonstrates mental health resilience, while Rutter et al. [28] found that about one-third of those who develop mental health difficulties do so only temporarily. Mental health resilience - relative resistance to psychopathology despite serious risk exposure [29] - observed in offspring of depressed parents indicates the presence of protective factors that may buffer parental depression-related risk effects. Identification of these protective factors and understanding of the processes through which individuals exposed to parental depression overcome experienced adversities would not only help to better understand mental health resilience and what distinguishes adaptive and maladaptive trajectories, but could also help to identify targets for evidence-based prevention and intervention strategies for those in need [30].

Despite being identified as a priority [11], evidence of protective factors that might explain mental health resilience in offspring of depressed parents is limited and systematic synthesis of these factors is still needed. Therefore, this systematic literature review will aim to identify parental/ caregivers', child, family, and social factors associated with mental health resilience in offspring exposed to parental depression. Considering the lack of a universally accepted definition of mental health resilience [31], this systematic review will also aim to identify how mental health resilience was conceptually and operationally defined in previously conducted studies. Since protective factors may differ for males and females, by offspring age or for different types of outcomes, the secondary aim will be to examine if there is enough evidence to support factors associated with mental health resilience that are specific to sex, developmental stage or mental health outcome in offspring exposed to parental depression.

\section{Methods}

\section{Design and registration}

The protocol for the proposed systematic literature review was developed according to the Preferred Reporting Items for Systematic Review and Meta-Analyses Protocols (PRISMA-P) guidelines [32]. For completed PRISMA-P checklist see Additional File 1. The protocol for this systematic review has been registered in the International Prospective Register of Systematic Reviews (PROSPERO) database (www.crd.york.ac.uk/PROSPERO, CRD42021229955). Any protocol amendments will be documented in the PROSPERO database.

\section{Objectives}

Following the PEO (i.e. Population, Exposure, Outcome) format for systematic reviews of association (aetiology) [33], the primary aim of this systematic literature review will be to systematically search and narratively synthesise studies examining parental depression characteristics (exposure) and protective 
factors (exposure) associated with mental health resilience (outcome) in offspring exposed to parental depression (population). Findings will be synthesised and critically assessed on the following questions:

1. How is mental health resilience defined in previously conducted studies?

2. Are parental depression characteristics and comorbid conditions associated with mental health resilience in their offspring?

3. Which child, family and social factors are associated with mental health resilience in offspring exposed to parental depression?

4. Are there protective factors that are specific to offspring's sex, developmental stage or different type of outcomes/ outcome domains?

The first question will be answered by systemising information from previous research on how mental health resilience was conceptually and operationally defined, which offspring outcome domains were examined to define resilience, and over what time period. The second question will systemise results of studies examining parental depression characteristics and comorbid conditions in relation to mental health resilience in their offspring. The third question, and the main focus of this review, will identify child, family, and social protective factors associated with mental health resilience in offspring exposed to parental depression. Finally, as a secondary aim, the review will examine if there is enough evidence to support sex-, developmental stage-, and outcome domain-specific factors associated with mental health resilience in offspring exposed to parental depression.

\section{Eligibility criteria}

Identified studies will be included in the proposed systematic review if they meet the following inclusion criteria:

1. The study is written in English and is published in a peer reviewed-journal.

2. The study is a primary research study examining factors associated with mental health resilience in child, adolescent, or adult offspring of depressed parents/caregivers, including both observational (i.e. prospective or retrospective cohort, case-control or cross-sectional) or randomised controlled trial (RCT) study designs. Factors defined as predictors or moderators will be eligible for inclusion.

3. At least one of the parents/ caregivers in the study meets clinical or research International Classification of Diseases (ICD) or Diagnostic and Statistical Manual of Mental Disorders (DSM) criteria for a depressive disorder, receives treatment for depressive disorder or reports depressive symptoms. Studies, including parents/ caregivers with comorbid conditions, using parental depressive disorder as a subgroup in the analyses or comparing its effects to other types of parental psychopathology will be eligible for inclusion. 
4. Study reports on mental health resilience in offspring. Studies defining mental health resilience as absence of psychopathology, absence of psychopathology and positive adjustment/ competence, and better than expected outcome will be eligible for inclusion.

Studies will be excluded if:

1. The study is written in another language than English and/ or published in a not peer-reviewed journal.

2. The study is a secondary research report (e.g. book chapter, review, letter, editorial, or commentary), grey literature, systematic review, meta-analysis, abstract or conference proceeding, or study employing other research designs than mentioned in the inclusion criteria.

3. Parents/ caregivers participating in the study are not receiving treatment for depression or their depressive disorder or symptoms are not assessed/ clearly reported. Studies combining/ merging parental depression with other mental health conditions will also be excluded.

4. Offspring mental health resilience is not clearly defined or reported.

\section{Search strategy}

A two-step search strategy will be performed to maximise the chances of identifying relevant studies. First, electronic searches will be performed by searching titles, abstracts, keywords, and subheadings for articles published from inception to 2021 in PsycINFO, Embase, MEDLINE, Web of Science Core Collection, and Cochrane Library. No filters will be applied. Then, additional articles will be identified by manually screening the references and citations of included studies.

Search terms that will be used in the proposed systematic literature review can be grouped into four key concepts: parents/caregivers, depression, offspring, and protective factors/ mental health resilience. Additionally, the exclusion terms will be used to eliminate secondary research reports, conference proceedings or studies employing research designs that do not meet eligibility criteria for this systematic review. The combination of terms that will be used in electronic searches is presented in Table 1. Terms and subheadings for the electronic searches were chosen on the basis of results of an initial scoping review, search terms used in previous narrative reviews on this topic, and consultation with university librarian and researchers with expertise in developmental psychopathology, mental health resilience and systematic literature reviews.

Insert Table 1 about here. 
Pilot testing of the search strategy was performed prior to registering the protocol to identify the utility of selected subject headings, terms and their combinations. Ten publications relevant to mental health resilience, including literature reviews and qualitative studies, were identified prior to the pilot search. Then, electronic searches were performed to examine if search strategy used can capture relevant publications identified and exclude those that employ research designs not eligible for inclusion (i.e. systematic reviews and qualitative studies). For selected publications and pilot search results see Additional File 2, while draft search strategy for one of the databases and full search strategy for each database are presented in Table 2 and Additional File 3, respectively.

Insert Table 2 about here.

\section{Screening procedure}

All search results will be exported from the database and imported into the reference management software EndNote ${ }^{\mathrm{TM}}$ and automatically and manually deduplicated by matching different combinations of author, title, and year of study. Following deduplication, references will be imported into systematic literature review software Rayyan [34] that will be used for title/abstract and full-text screening, documentation of screening progress, and reasons for exclusion. First, titles and abstracts will be screened to identify publications for full-text retrieval. If at this stage, reviewers are unsure about the eligibility of the study, it will be included in the full-text screening stage. Then, full texts of potentially eligible studies will be assessed against previously mentioned inclusion and exclusion criteria. Discrepancies between reviewers, if any, will be resolved during consensus meetings with a senior researcher (SC). Finally, additional articles will be identified by screening references and citations of studies included in the systematic review. The literature search and deduplication will be performed by the first reviewer only (EP), while title/ abstract and full-text screening of studies identified by electronic searchers and identification of additional papers will be independently done by both first and second (JMM) reviewers. Reviewers will be blinded to the other's decisions during this process.

\section{Data Extraction}

Two separate data extraction forms for observational studies and RCTs will be developed following Preferred Reporting Items for Systematic Review and Meta-Analyses (PRISMA) [32] and Cochrane [35] guidelines. Extracted data will be compiled in an Excel sheet. For observational studies, the form will include information on study characteristics (i.e. first author and date, country of study, study design, years of follow-up, study aims), population characteristics (i.e. sample size, offspring age and sex, and 
time of exposure to parental depression), exposure characteristics (i.e. depressed parent's sex and age, type and characteristics of parental depressive disorder, comorbid conditions, and assessment and diagnostic instruments used), outcome (i.e. the definition of mental health resilience, offspring's mental health/ resilience outcome result, the timing of outcome measurement, and assessment and diagnostic instruments used), comparison group (if any), protective factors, a measure of association, p-value, covariates, if any, used in the model, main findings, limitations, and risk of bias assessment outcome.

For RCTs, aforementioned study characteristics, population characteristics (i.e. depressed parent's age and sex, type and (baseline) characteristics of parental depressive disorder, comorbid conditions, and assessment and diagnostic instruments used), intervention characteristics (i.e. type of intervention, timing, duration, and delivery of intervention), comparison group, outcome characteristics (i.e. the definition of mental health resilience, parent's/caregiver's and offspring's mental health outcome, the timing of assessment, and assessment and diagnostic instruments used), a measure of association, pvalue, main findings, limitations, and risk of bias assessment outcome will be reported.

Data extraction will be performed by the first reviewer only. The second reviewer will independently validate at least $20 \%$ of the data extracted.

\section{Risk of Bias Assessment}

Risk of bias assessments will be performed separately for observational studies and RCTs. In line with recommendations, no observational studies will be excluded based on the risk of bias assessment outcome [36]. For observational studies, Joanna Briggs Institute (JBI) critical appraisal checklists for cohort, case-control, and cross-sectional studies will be used [37]. These critical appraisal tools consider comparability of groups, appropriateness of exposure and outcome assessments, identification and handling of confounding factors, and appropriateness of statistical analyses used. Each domain will be evaluated as being at a high, low, or unclear risk of bias or by selecting a not applicable option.

The Revised Cochrane risk of bias tool for randomised trials (RoB 2) will be used for RCTs [38]. The risk of bias arising from the randomisation process, deviations from the intended intervention, missing outcome data, measurement of outcome data, and selection of the reported results will be judged by selecting one of five options (Yes; Probably Yes; Probably No; No; No Information). 
The risk of bias assessment will be performed by the first reviewer only, but as a reliability check, the second reviewer will independently perform risk of bias assessment of at least $20 \%$ of the studies. Cohen's Kappa coefficient will be calculated to assess inter-rater agreement between two reviewers.

\section{Data Synthesis}

Due to expected heterogeneity across studies in terms of definitions of mental health resilience, protective factors, study designs (e.g. RCTs, observational), and outcome assessment tools, meta-analysis will not be attempted in this systematic literature review. Therefore, a narrative synthesis of data extracted from the selected studies will be performed. First, the descriptive characteristics of the included studies will be reported. These will include countries of studies, years of publication, study designs, the risk of bias assessment outcomes, population description (offspring sex, age, and time of exposure to parental depression), and exposure description (parental depression chronicity, severity, and time of onset). Then, to address the primary and secondary research questions of this review, the main findings will be presented in six major sections:

1. Definition of mental health resilience in previously conducted studies.

2. Parental/ caregiver factors associated with offspring mental health resilience, such as (improvement in) parental depression characteristics and/ or (absence of) comorbid conditions.

3. Family factors associated with mental health resilience.

4. Social factors associated with mental health resilience.

5. Child factors associated with mental health resilience (i.e. biological, psychological, and lifestyle factors).

6. Sex-, developmental stage-, and outcome- specific factors associated with mental health resilience.

Considering that depressive symptoms may reflect general distress rather than depressive disorders [39, 40], results of studies reporting depressive symptoms and depressive disorders in parents/ caregivers will be reported separately.

\section{Discussion}

This systematic review will define mental health resilience in the parental depression context and identify parental/ caregivers', child, family, and social factors associated with mental health resilience in offspring exposed to parental depression. The findings will help better understand mental health resilience and factors associated with it and identify future research directions. The findings are also expected to identify modifiable targets for evidence-based prevention and intervention strategies that could contribute 
to the discontinuity of intergenerational transmission of psychopathology and improve the lives of both depressed parents and their offspring.

\section{Abbreviations}

ADHD

attention deficit hyperactivity disorder; PRISMA-P:Preferred Reporting Items for Systematic Review and Meta-Analyses Protocols; PROSPERO:International Prospective Register of Systematic Reviews; PEO:systematic review question format outlining population or group at risk, exposure, and outcome; RCT:randomized controlled trial; ICD:International Classification of Diseases; DSM:Diagnostic and Statistical Manual of Mental Disorders; PRISMA:Preferred Reporting Items for Systematic Review and Meta-Analyses; RoB 2:revised Cochrane risk of bias tool for randomised trials.

\section{Declarations}

\section{Ethics approval and consent to participate}

This systematic literature review does not require ethics approval.

\section{Consent for publication}

All authors have provided consent for publication.

\section{Availability of data and materials}

Not applicable.

\section{Competing interests}

The authors declare that they have no competing interests.

\section{Funding}

EP is supported by a grant from Mental Health Research UK and the Schizophrenia Research Fund. JM is supported by the São Paulo Research Foundation (FAPESP) (grant number: 2017/22723-5). GH is supported by a Sir Henry Wellcome Postdoctoral Fellowship (grant number: 209138/Z/ 17/Z). The sponsors had no role in the development of this protocol. 


\section{Authors' contributions}

EP is the guarantor and drafted the protocol. All authors contributed to the conceptualisation and development of the selection criteria and search strategy, and the strategy for data extraction, risk of bias assessment, and data synthesis. All authors approved the final manuscript before submission.

\section{Acknowledgements}

We would like to express our gratitude to Mari Ann Hilliar, librarian at the Health Library, Cardiff University for peer review of our search strategy. This research was funded in whole, or in part, by the Wellcome Trust [209138/Z/ 17/Z]. For the purpose of Open Access, the author has applied a CC BY public copyright license to any Author Accepted Manuscript version arising from this submission.

\section{References}

1. American Psychiatric Association. Diagnostic and statistical manual of mental disorders: DSM-5. 5th ed. ed. Arlington, Va.: Arlington, Va. : American Psychiatric Association; 2013.

2. Abate KH, Abebe Z, Abil OZ, Afshin A, Ahmed MB, Alahdab F, et al. Global, regional, and national incidence, prevalence, and years lived with disability for 354 diseases and injuries for 195 countries and territories, 1990-2017: a systematic analysis for the Global Burden of Disease Study 2017. The Lancet (British edition). 2018;392(10159):1789-858.

3. Chesney E, Goodwin GM, Fazel S. Risks of all-cause and suicide mortality in mental disorders: a meta-review. World psychiatry. 2014;13(2):153-60.

4. Swendsen JD, Merikangas KR. The comorbidity of depression and substance use disorders. Clin Psychol Rev. 2000;20(2):173-89.

5. Hasler G, Pine DS, Kleinbaum DG, Gamma A, Luckenbaugh D, Ajdacic V, et al. Depressive symptoms during childhood and adult obesity: the Zurich Cohort Study. Molecular psychiatry. 2005;10(9):84250 .

6. Whooley MA, Wong JM. Depression and Cardiovascular Disorders. Ann Rev Clin Psychol. 2013;9(1):327-54.

7. Kupferberg A, Bicks L, Hasler G. Social functioning in major depressive disorder. Neurosci Biobehav Rev. 2016;69:313-32.

8. Beardselee WR, Versage EM, Giadstone TRG. Children of Affectively III Parents: A Review of the Past 10 Years. J Am Acad Child Adolesc Psychiatry. 1998;37(11):1134-41.

9. Weissman MM, Wickramaratne P, Gameroff MJ, Warner V, Pilowsky D, Kohad RG, et al. Offspring of Depressed Parents: 30 Years Later. Am J Psychiatry. 2016;173(10):1024-32. 
10. Abel KM, Hope H, Swift E, Parisi R, Ashcroft DM, Kosidou K, et al. Prevalence of maternal mental illness among children and adolescents in the UK between 2005 and 2017: a national retrospective cohort analysis. The Lancet Public health. 2019;4(6):e291-300.

11. Weissman MM. Children of Depressed Parents-A Public Health Opportunity. JAMA psychiatry. (Chicago III). 2016;73(3):197-8.

12. Rice F, Harold G, Thapar A. The genetic aetiology of childhood depression: a review. J Child Psychol Psychiatry. 2002;43(1):65-79.

13. Thapar A, Collishaw S, Pine DS, Thapar AK. Depression in adolescence. The Lancet. 2012;379(9820):1056-67.

14. Weissman MM, Wickramaratne P, Nomura Y, Warner V, Pilowsky D, Verdeli H. Offspring of Depressed Parents: 20 Years Later. Am J Psychiatry. 2006;163(6):1001-8.

15. Tully CE, lacono GW, McGue M. An Adoption Study of Parental Depression as an Environmental Liability for Adolescent Depression and Childhood Disruptive Disorders. Am J Psychiatry. 2008;165(9):1148-54.

16. Mars B, Collishaw S, Smith D, Thapar A, Potter R, Sellers R, et al. Offspring of parents with recurrent depression: Which features of parent depression index risk for offspring psychopathology? J Affect Disord. 2011;136(1):44-53.

17. Sellers R, Harold GT, Elam K, Rhoades KA, Potter R, Mars B, et al. Maternal depression and cooccurring antisocial behaviour: testing maternal hostility and warmth as mediators of risk for offspring psychopathology. J Child Psychol Psychiatry. 2014;55(2):112-20.

18. Rehman US, Gollan J, Mortimer AR. The marital context of depression: Research, limitations, and new directions. Clin Psychol Rev. 2008;28(2):179-98.

19. Downey G, Coyne JC. Children of Depressed Parents: An Integrative Review. Psychological bulletin. 1990;108(1):50-76.

20. Gladstone BM, Boydell KM, McKeever P. Recasting research into children's experiences of parental mental illness: Beyond risk and resilience. Social science \& medicine (1982). 2006;62(10):2540-50.

21. Murray L, Halligan SL, Adams G, Patterson P, Goodyer IM. Socioemotional development in adolescents at risk for depression: The role of maternal depression and attachment style. Dev Psychopathol. 2006;18(2):489-516.

22. Kane P, Garber J. The relations among depression in fathers, children's psychopathology, and fatherchild conflict: a meta-analysis. Clin Psychol Rev. 2004;24(3):339-60.

23. Mahedy L, Harold GT, Maughan B, Gardner F, Araya R, Bevan Jones R, et al. Resilience in high-risk adolescents of mothers with recurrent depressive disorder: the contribution of fathers. Journal of adolescence. 2018;65:207-18.

24. Vakrat A, Apter-Levy Y, Feldman R. Sensitive Fathering Buffers the Effects of Chronic Maternal Depression on Child Psychopathology. Child Psychiatry Hum Dev. 2018;49(5):779-85. 
25. Branje S, Geeraerts S, de Zeeuw EL, Oerlemans AM, Koopman-Verhoeff ME, Schulz S, et al. Intergenerational transmission: Theoretical and methodological issues and an introduction to four Dutch cohorts. Developmental cognitive neuroscience. 2020;45:100835-.

26. Merikangas KR, Spiker DG. Assortative mating among in-patients with primary affective disorder. Psychological medicine. 1982;12(4):753-64.

27. Collishaw S, Hammerton G, Mahedy L, Sellers R, Owen MJ, Craddock N, et al. Mental health resilience in the adolescent offspring of parents with depression: a prospective longitudinal study. The Lancet Psychiatry. 2016;3(1):49-57.

28. Rutter M, Quinton D. Parental psychiatric disorder: effects on children. Psychological medicine. 1984;14(4):853-80.

29. Rutter M. Implications of Resilience Concepts for Scientific Understanding. Ann N Y Acad Sci. 2006;1094(1):1-12.

30. Rutter M. Annual Research Review: Resilience - clinical implications. J Child Psychol Psychiatry. 2013;54(4):474-87.

31. Aburn G, Gott M, Hoare K. What is resilience? An Integrative Review of the empirical literature. Journal of advanced nursing. 2016;72(5):980-1000.

32. Moher D, Liberati A, Tetzlaff J, Altman DG. Preferred reporting items for systematic reviews and meta-analyses: the PRISMA statement. BMJ. 2009;339(7716):332-6.

33. Moola S, Munn Z, Sears K, Sfetcu R, Currie M, Lisy K, et al. Conducting systematic reviews of association (etiology): The Joanna Briggs Institute's approach. Int J Evid Based Healthc. 2015;13(3):163-9.

34. Ouzzani M, Hammady H, Fedorowicz Z, Elmagarmid A. Rayyan-a web and mobile app for systematic reviews. Systematic reviews. 2016;5(1):210-.

35. Higgins JPT, Green S. Cochrane Handbook for Systematic Reviews of Interventions. 1. Aufl. 1st ed. ed. Hoboken: Hoboken: Wiley; 2008.

36. Dekkers OM, Vandenbroucke JP, Cevallos M, Renehan AG, Altman DG, Egger M. COSMOS-E: Guidance on conducting systematic reviews and meta-analyses of observational studies of etiology. PLoS Med. 2019;16(2):e1002742-e.

37. Moola S, Munn Z, Tufanaru C, Aromataris E, Sears K, Sfetcu R, Currie M, Qureshi R, Mattis P, Lisy K, Mu P-F. Chapter 7: Systematic reviews of etiology and risk. In: Aromataris E, Munn Z, editors. JBI Manual for Evidence Synthesis. JBI, 2020. https://synthesismanual.jbi.global. Accessed 20 Jan 2021.

38. Higgins JPT, Sterne JAC, Savović J, Page MJ, Hróbjartsson A, Boutron I, Reeves B, Eldridge S. A revised tool for assessing risk of bias in randomized trials In: Chandler J, McKenzie J, Boutron I, Welch V, editors. Cochrane Methods. Cochrane Database of Systematic Reviews 2016, 10(Suppl 1).

39. Coyne JC. Self-Reported Distress: Analog or Ersatz Depression? Psychological bulletin. 1994;116(1):29-45. 
40. Lewinsohn PM, Gotlib IH, Seeley JR. Adolescent psychopathology. IV: Specificity of psychosocial risk factors for depression and substance abuse in older adolescents. J Am Acad Child Adolesc Psychiatry. 1995;34(9):1221-9.

\section{Tables}

Table 1. Subject headings and search terms that will be used in the proposed systematic review. 


\begin{tabular}{|c|c|c|c|}
\hline $\begin{array}{l}\text { Key } \\
\text { concept }\end{array}$ & Subject headings & \multirow[t]{2}{*}{ OR } & Terms \\
\hline $\begin{array}{l}\text { Parents/ } \\
\text { Caregivers }\end{array}$ & $\begin{array}{l}\text { caregiver OR caregivers OR } \\
\text { parent OR parents }\end{array}$ & & $\begin{array}{l}\text { caregiver* OR parent* OR } \\
\text { maternal OR paternal OR } \\
\text { mother* OR father* }\end{array}$ \\
\hline \multicolumn{4}{|c|}{ AND } \\
\hline Depression & $\begin{array}{l}\text { depression OR mood disorder OR mood } \\
\text { disorders OR affective disorders OR major } \\
\text { depression }\end{array}$ & OR & $\begin{array}{l}\text { depress* OR affective } \\
\text { disorder* OR mood } \\
\text { disorder* }\end{array}$ \\
\hline \multicolumn{4}{|c|}{ AND } \\
\hline Offspring & child OR offspring & OR & $\begin{array}{l}\text { offspring* OR child* OR } \\
\text { son* OR daughter* }\end{array}$ \\
\hline \multicolumn{4}{|c|}{ AND } \\
\hline $\begin{array}{l}\text { Protective } \\
\text { factors/ } \\
\text { Mental } \\
\text { health } \\
\text { resilience }\end{array}$ & $\begin{array}{l}\text { psychological resilience OR resilience, } \\
\text { psychological OR resilience } \\
\text { (psychological) OR protective factors }\end{array}$ & OR & $\begin{array}{l}\text { resilien* OR protect* OR } \\
\text { buffer* OR mitigat* risk } \\
\text { OR optimi* outcome* OR } \\
\text { adapt* functioning OR } \\
\text { thriv* OR positive } \\
\text { adaptation }\end{array}$ \\
\hline \multicolumn{4}{|c|}{ NOT } \\
\hline $\begin{array}{l}\text { Exclusion } \\
\text { terms }\end{array}$ & $\begin{array}{l}\text { "review” OR "literature review” OR } \\
\text { “systematic review” OR metaanalysis OR } \\
\text { meta-analysis OR qualitative methods OR } \\
\text { qualitative research OR conference paper } \\
\text { OR congresses }\end{array}$ & OR & $\begin{array}{l}\text { review* OR comment* OR } \\
\text { letter OR meta-analysis } \\
\text { OR metaanalysis OR } \\
\text { editorial OR conference } \\
\text { publication OR qualitative }\end{array}$ \\
\hline
\end{tabular}

Table 2. Draft search strategy for one of the databases. 


\begin{tabular}{|c|c|}
\hline Database & Terms \\
\hline $\begin{array}{l}\text { Ovid } \\
\text { MEDLINE }\end{array}$ & 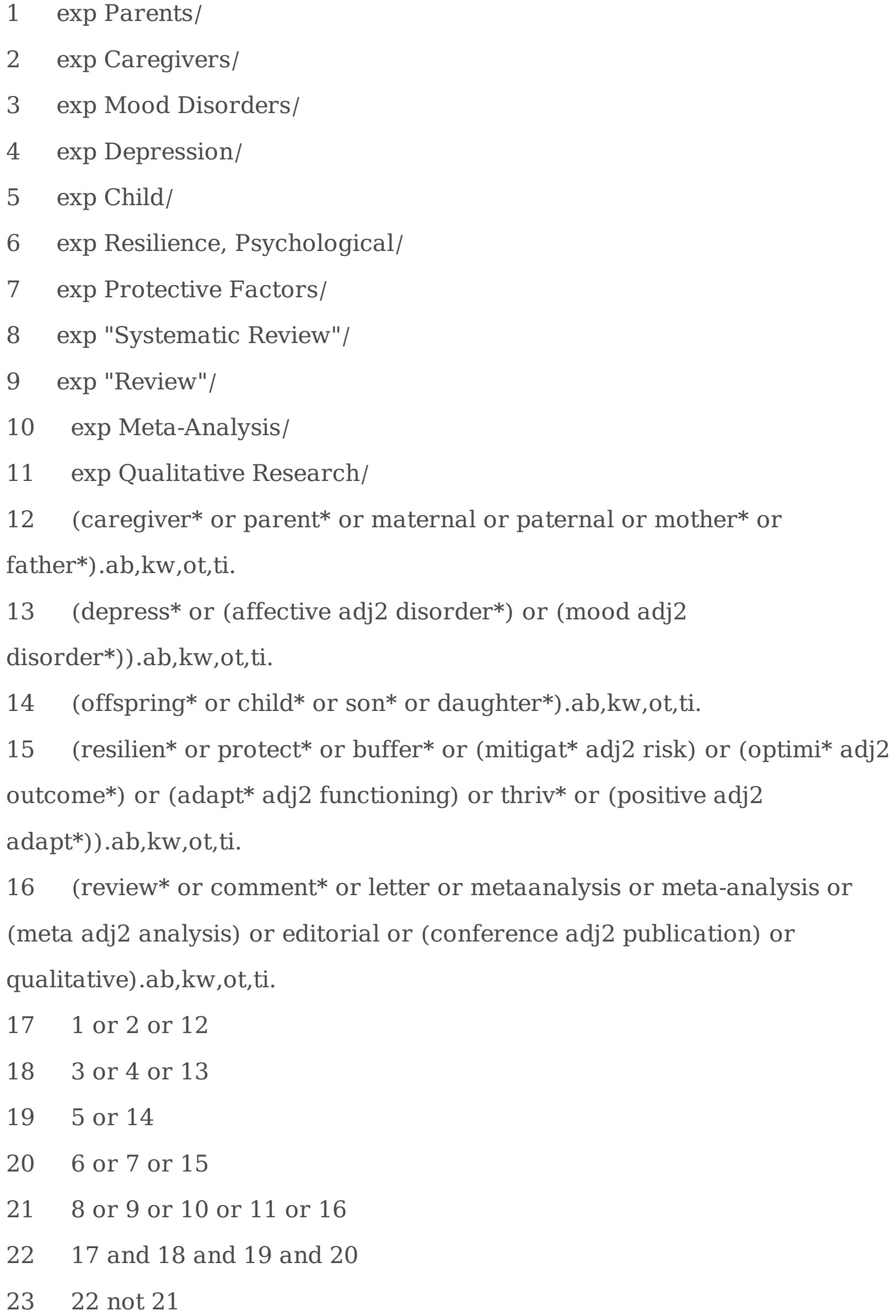 \\
\hline
\end{tabular}




\section{Supplementary Files}

This is a list of supplementary files associated with this preprint. Click to download.

- Additionalfile1.PRISMAPchecklist.pdf

- Additionalfile2.Pilotsearchresults.pdf

- Additionalfile3.Searchstrategyforeachdatabase.pdf 